Fiori dell'anima. La simbologia dei fiori nell'immaginario religioso Flowers of the soul. The symbolism of flowers in the religious imagination a cura di Marco Papasidero e Francesco Galofaro

\title{
Come vestono i gigli del campo Trasformazioni dell'immaginario floreale nei primi secoli cristiani
}

Graziano Lingua

Università di Torino, IT

graziano.lingua@unito.it

\begin{abstract}
The essay analyzes the debate about use of flowers in the Early Christianity identifying two principal attitudes of the Fathers of the Church. Initially there is a negative attitude towards flowers that is related with the critique of the pagan idolatry (Tertullian, Minucius Felis, Clemens of Alexandria). Along side to this refusal of pagan practises gradually grows a more positive floreal imaginary that valorizes the beauty of flowers as symbol of the presence of God and his divine providence. Analyzing some patristical comments to the famous Gospel passage of Mt 6 about "lilies of the fild", the autor shows that this new imaginary is related to a more broader transfomation of Christian sensibility towards creatural beauty that involves not only flowers but also the sacred images.
\end{abstract}

\section{Keywords}

Floreal imagery; Fathers of the Church; Early Christianity; Idolatry; Crowns; Gardens

\section{Sommario/Content}

1. Introduzione

2. Tutto comincia con un giardino senza fiori

3. Contro le corone e l'uso pagano dei fiori

4. I gigli del campo e la cura di Dio

5. Conclusione

Bibliografia 


\section{Dcula ${ }^{23}$}

Vol 21, No 23 (July 2020) • DOI: 10.12977/ocula2020-29

Graziano Lingua | Come vestono i gigli del campo. Trasformazioni dell'immaginario floreale nei primi secoli cristiani

\section{Introduzione}

Nel suo libro La cultura dei fiori, Jack Goody analizza l'atteggiamento della chiesa cristiana dei primi secoli in un capitolo significativamente intitolato Il declino della cultura dei fiori in Europa (Goody 1993: 91-130). Dopo aver ricostruito l'importanza attribuita alla cultura dei fiori nelle civiltà euroasiatiche (che risalta particolarmente, se la si confronta con la quasi completa assenza di fiori nella cultura africana), e dopo aver descritto con dovizia di particolari i giardini mesopotamici, la coltivazione dei fiori nella valle del Nilo, la loro diffusione nella vita religiosa e civile dei greci e dei romani, Goody ci mette di fronte a un fatto indiscutibile: con la caduta di Roma tutto questo tripudio floreale sembra essere ormai tramontato (Goody 1993: 91). La crisi delle strutture economiche e politiche, che avevano fatto la grandezza dell'Impero, aveva trascinato via con sé anche i giardini, la coltivazione dei fiori, il loro utilizzo pubblico e la loro commercializzazione, tutti elementi integranti di una agricoltura avanzata, ormai entrata in crisi con il movimento migratorio su larga scala dei cosiddetti popoli barbari (Tosco 2018: 103-107). La causa di questo drastico fenomeno di declino della cultura floreale non va però cercata - e questa è la tesi specifica di Goody - nei soli fattori materiali perché

la cultura dei fiori avrebbe potuto perdurare in una forma o essere riportata in vita in un'altra, se importanti personalità dell'antica Chiesa cristiana non avessero attivamente scoraggiato l'uso di pratiche pagane in molte circostanze religiose. (Goody 1993: 92)

Tra queste pratiche vi era sicuramente quella di offrire fiori agli dei e ai defunti, quella di ornare di ghirlande le statue delle divinità o ancora di indossare corone di foglie e fiori, tutte usanze che facevano parte integrante non solo dei riti religiosi, ma anche di quelli pubblici.

È certamente vero che i fiori furono trascinati dai Padri della Chiesa nella polemica anti-idolatrica e che le critiche ai sacrifici alle divinità vennero estese anche ad essi e ai giardini in cui venivano coltivati (Goody 1993: 107), ma l'obiettivo di questa polemica non erano i fiori in sé, quanto piuttosto il loro diretto collegamento con le pratiche cultuali pagane. I fiori erano quindi la cifra di una posta simbolica più generale che coinvolgeva la natura stessa del cristianesimo e la sua concezione del divino. Essi si trovarono coinvolti nei primi secoli in una trasformazione culturale di grande portata che toccava direttamente lo sforzo operato dal cristianesimo per differenziarsi rispetto al paganesimo e dare forma a una nuova sensibilità religiosa fondata sull'interiorità del culto e su una forte scelta morale individuale. Peraltro non bisogna dimenticare che il cristianesimo era nato in seno all'ebraismo, una religione che non era propensa all'uso di fiori nelle proprie liturgie e che si era distinta dai popoli confinanti per l'austerità rituale e per una visione spirituale che condannava come idolatrica ogni forma di venerazione di realtà naturali. Giustamente Goody nota come l'atteggiamento negativo dell'ebraismo verso i fio- 


\section{Dcula ${ }^{23}$}

Vol 21, No 23 (July 2020) • DOI: 10.12977/ocula2020-29

Graziano Lingua | Come vestono i gigli del campo. Trasformazioni dell'immaginario floreale nei primi secoli cristiani

ri si colleghi alla condanna delle immagini che ritroviamo nel secondo comandamento (Es 20,3-4) (Goody 1993: 55), perché entrambe pongono l'accento sul fatto che l'esteriorità visibile è incompatibile con un Dio come Yhwh, che nessuno può vedere in volto restando in vita (Es 33,20).

Questa dimensione della visibilità di Dio e della sua manifestazione nel mondo sarà proprio la matrice su cui poi maturerà nel cristianesimo un diverso atteggiamento, questa volta positivo, che accomunerà le immagini religiose e i fiori. Anche in questo caso la sorte dei fiori sarà determinata da una trasformazione ideologica che si fonderà sulla nuova sensibilità per la presenza visibile di Dio nel mondo grazie all'incarnazione di suo Figlio e per la cura provvidenziale che questo Dio ha nei confronti degli uomini. Già nella letteratura patristica infatti si trovano i germi di una valorizzazione della funzione estetico-simbolica dei fiori e i primi sintomi di questo immaginario floreale positivo sono significativamente imparentati con il percorso di legittimazione delle immagini religiose perché toccano entrambe il modo con cui il cristianesimo intende la mediazione tra la trascendenza e l'immanenza di Dio. Le vicende ambivalenti che accomunano i fiori e le immagini si intrecciano infatti alle difficoltà teologiche che il primo cristianesimo ha avuto a riconoscere e valorizzare la nuova concezione del divino implicata nel messaggio e nella vicenda di Gesù di Nazareth (Kelly 1984; Prestige 2018; Wilken 2006), che riarticolava il senso stesso della critica anti-idolatrica presente nell'ebraismo. Lo sforzo di negoziare la trascendenza di un Dio invisibile ereditato dall'Antico Testamento con la sua presenza nella storia e nella bellezza della creazione richiedeva di riformulare complessivamente non solo le forme cultuali, ma anche e più profondamente i presupposti teologici che le rendevano o meno legittime. Di tale rielaborazione Goody ricostruisce nel proprio libro prevalentemente gli esiti medioevali e individua nel XII secolo il momento in cui avviene un esplicito "ritorno" della cultura dei fiori nel cristianesimo (Goody 1993: 159-204). In queste brevi note vorrei mostrare come già nel primo cristianesimo, accanto alla critica nei confronti dei fiori, cominci a emergere un immaginario positivo che getterà le basi dello sviluppo del simbolismo dei fiori che grande importanza avrà nella successiva pratica liturgica cristiana $\mathrm{e}$ nell'intera cultura occidentale.

\section{Tutto comincia con un giardino senza fiori}

Com'è noto il canone biblico comincia con la descrizione di un giardino. Quando Yhwh pianta questo giardino in Eden lo riempie «di ogni sorta di alberi graditi alla vista e buoni da mangiare» (Gen 2,9), ma non vi mette dei fiori. Il fatto è ancora più strano se si pensa che l'Eden di cui si parla nel secondo capitolo di Genesi ha chiare parentele con i giardini medio-orientali, in cui era invece normale che accanto a piante da frutto ci fosse un'orticoltura estetica. Tale parentela è evidente se si considera il fatto che nella traduzione greca dei LXX il termine ebraico per indicare giardino, gan, viene reso con la parola $\pi \alpha \rho \alpha ́ \delta \varepsilon \varepsilon \sigma o \zeta$, che evidenzia il legame con il "paradiso" persiano (pairi-daeza), un tipo di giardino chiuso di cui si sono trovate tracce in diverse 


\section{Decula ${ }^{23}$}

Vol 21, No 23 (July 2020) • DOI: 10.12977/ocula2020-29

Graziano Lingua | Come vestono i gigli del campo. Trasformazioni dell'immaginario floreale nei primi secoli cristiani

residenze reali della dinastia achemenide. ${ }^{1}$ Certo, se si confronta l'Eden con quello che potevano essere i mitici giardini pensili di Babilonia, considerati una delle sette meraviglie del mondo (cfr. Finkel 2008: 109-111), o con i paradisi di Ciro il Grande di cui parla in più occasioni Senofonte, ${ }^{2}$ si può rimanere stupiti che il testo biblico si limiti a parlare di alberi e non annoveri altre piante e fiori. Lo stupore diminuisce se si considera che le piante che vengono citate nell'Antico Testamento, fatte alcune eccezioni tra cui si possono ricordare l'abbondanza di fiori presenti nel Cantico dei Cantici (gigli, narcisi, zafferani, fiori di melograno),${ }^{3}$ sono il più delle volte erbe aromatiche ed edibili, ritenute più importanti per la loro efficacia che per il loro aspetto. ${ }^{4} \mathrm{Di}$ fiori si parla rispetto al tabernacolo mosaico (Es 25, 31-36; 37, 17-22; Nm 8,4) e agli allestimenti del tempio di Salomone (1 Re 6-7), come la verga di Aronne fiorì

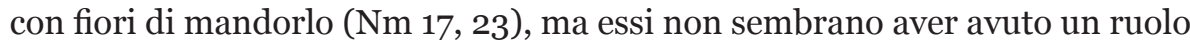
religioso così consistente come nelle culture limitrofe. Le ricorrenze dedicate ai fiori nei libri profetici e sapienziali sottolineano spesso la loro fragile caducità. «Come l'erba sono i giorni dell'uomo, come il fiore del campo, così egli fiorisce. Lo investe il vento e più non esiste», canta il salmista (Sal 104, 15-16). Accanto alla caducità umana la letteratura biblica accosta poi i fiori al giudizio degli orgogliosi e degli empi che come i fiori hanno un momento di gloria e poi appassiscono e seccano (Is 28,1-4; $\mathrm{Na} \mathrm{1,4).}$

L'ebraismo biblico non è tuttavia indifferente alla bellezza dei fiori e alla loro potenza metaforica per esprimere la rinascita e la speranza, come avviene ad esempio nell'oracolo di Is 35,1-2: «Si rallegrino il deserto e la terra arida, esulti e fiorisca la steppa. Come fiore di narciso fiorisca». Resta il fatto che nella letteratura biblica i fiori non acquisiranno mai l'importanza che viene attribuita loro dalle principali culture antiche. La motivazione di questa attitudine va cercata nella specifica prassi cultuale dell'ebraismo e nel fatto che i fiori erano facilmente associati a pratiche idolatriche. Bisogna poi tenere in conto che essi erano direttamente collegati ai piaceri degli occhi e della vista che il racconto di Genesi collega con il peccato di Eva (Gen 3,6). Il comandamento contro le immagini e l'idolatria (Es 20,3-4; Dt 5,8) inscriveva in questa religione un profondo sospetto verso tutto ciò che avesse a che vedere con la venerazione di realtà materiali o con la loro rappresentazione visiva. Non è un caso allora che, alla fine del II secolo d.C., Minucio Felice nel suo dialogo Octavius, si riferisca implicitamente al divieto ebraico delle immagini per giustificare il fatto che anche i cristiani, come gli ebrei, non hanno bisogno di «formarsi un'immagine materiale di Dio» (Octavius, 32, 1; tr. it in Menozzi 1995: 71), perché la loro anima è l'unico tempio degno di tale nome.

1 Probabilmente i traduttori della LXX dovevano però avere in mente i giardini di epoca ellenistica (cfr. J. Bremmer 1999: 18-19).

2 Si veda ad esempio il brano che Senofonte dedica ai paradisi in Oeconomicus, I, 20-22 (tr. it. Senofonte 2000: 103). Sui paradisi del periodo achemenide si veda Tuplin (1996: 80-131).

3 Cfr. ad esempio: Ct 2,1; 2, 12.13; 4,13-14; 6,2-3; 6, 11; 7,13.

4 Sul ruolo dei fiori nella letteratura biblica si veda in particolare la voce "fiori" in Ryden, Wilhot, Longman (2006: 540-543), a cui mi sono ampiamente riferito. 


\section{Dcula ${ }^{23}$}

Vol 21, No 23 (July 2020) • DOI: 10.12977/ocula2020-29

Graziano Lingua | Come vestono i gigli del campo. Trasformazioni dell'immaginario floreale nei primi secoli cristiani

Quanto è meglio - si legge nel dialogo - dedicargli la nostra anima e consacrare a Lui la parte più eletta di noi. Perché offrirgli vittime e sacrifici? [...] Quello che gli dobbiamo consacrare è un animo retto, un cuore puro, una sincera coscienza. (Octavius, 32, 1; tr. it in Menozzi 1995: 71)

L'ebraismo consegna al primo cristianesimo una sensibilità religiosa imperniata sull'ascolto della parola e non sulla vista di immagini, e quindi poco propensa al culto esteriore. Per distinguersi dagli altri popoli medio-orientali dell'epoca Israele aveva dovuto prendere le distanze dalle loro pratiche cultuali e segnare lo iato tra la fede in un Dio unico rispetto al variegato pantheon delle divinità circostanti. In questo sforzo di differenziazione erano coinvolti molti riti, in cui si faceva ampio uso di fiori, e i primi cristiani non potevano essere favorevoli ad essi, tanto più che il Nuovo Testamento non offriva nessun appiglio per un uso cultuale dei fiori.

\section{Contro le corone e l'uso pagano dei fiori}

Che l'obiettivo della critica cristiana ai fiori fosse primariamente l'uso cultuale che ne facevano i pagani lo si desume dalla polemica che molti Padri della Chiesa portano avanti contro le corone floreali. Queste corone venivano indossate durante i culti sacrificali sia dai sacerdoti, sia dai partecipanti al rito e poste intorno al collo delle vittime animali destinate ad essere immolate. Con corone di foglie e di fiori venivano adornate le statue degli dei o dei lari e l'incoronazione era parte del rito consacratorio delle immagini di divinità. Esse erano adoperate anche dagli indovini durante le divinazioni, indossate da poeti e magistrati (Baus, 2-36). Proprio per questo significato rituale nei primi secoli cristiani esse assurgono quindi a emblema della critica ai culti pagani e sono direttamente collegate con quella che era considerata l'idolatria, il culto di falsi dei. È così che alla condanna dei sacrifici agli dèi si accomuna anche la condanna delle offerte floreali e dell'uso cerimoniale delle corone.

Il testo più noto di questa polemica è certamente il De corona di Tertulliano. L'opera ha come occasione l'esaltazione del coraggio di un soldato romano della III Legio Augusta che a Lambesi nel 211 rifiutò di indossare la corona militare prima di ricevere il donativum alla morte dellimperatore Settimio Severo. Alla richiesta del motivo per cui egli si comportava così il soldato rispose che era cristiano e che una simile pratica era incompatibile con la sua fede (De corona, I, 2; tr. it. in Tertulliano 1980: 137). A partire da questo episodio Tertulliano si concentra per ben undici capitoli (De corona, II-XII; tr. it. in Tertulliano 1980: 149-204) sui motivi che devono spingere i cristiani a evitare l'uso delle corone. Scorrendo il dispiegarsi del suo ragionamento sul perché sia giusto che un cristiano non indossi corone di fiori e di alloro ci si imbatte in quelli che sono i motivi principali dell'immaginario negativo nei confronti dell'uso dei fiori della chiesa primitiva. Innanzitutto, come già dicevamo, l'aspetto che li rende sospetti è il fatto che essi sono immediatamente posti in relazione con gli dei pagani e con il culto a essi attribuito. Il loro im- 


\section{Dcula ${ }^{23}$}

Vol 21, No 23 (July 2020) • DOI: 10.12977/ocula2020-29

Graziano Lingua | Come vestono i gigli del campo. Trasformazioni dell'immaginario floreale nei primi secoli cristiani

piego si collega quindi alla partecipazione a cerimoniali pubblici che troppo spesso secondo Tertulliano i cristiani accolgono con facile accondiscendenza, ma che non appartengono alla tradizione della chiesa, né sono presenti nell'Antico Testamento.

$\mathrm{Al}$ di là di questo motivo strettamente teologico Tertulliano adduce anche una ragione filosofica più generale. I fiori non sono stati creati per essere portati sulle teste, ma per soddisfare il senso della vista e dell'olfatto e quindi reciderli per indossarli è un atteggiamento contro natura, mentre è legittimo reciderli per farne dei mazzi.

I fiori, anche se sono intrecciati, annodati, legati con fili o con rametti di giunco, vanno trattati come se fossero all'aria aperta sui campi, cioè come cose fatte per essere guardate e per sentirne il profumo [...] Ma se te li metti in testa, che sensazione possono mai offrire quei fiori? Se mi metto una corona di fiori in testa sento solo qualcosa che mi avvolge le tempie, i colori non li vedo, il profumo non lo sento e la bellezza dei fiori non posso ammirarla. (De corona, V, 4; tr. it. in Tertulliano 1980: 161)

Curiosamente ritroviamo lo stesso argomento anche nel già citato Octavius di Minucio Felice. In questo dialogo Cecilio, che impersona la posizione del paganesimo, critica i cristiani perché rifiutano l'uso di fiori per abbellire il corpo e onorare le sepolture e Ottavio risponde che i cristiani raccolgono volentieri i fiori per il loro colore e per i loro profumi e ne fanno anche ghirlande da mettere al collo, ma non li mettono sul loro capo perché sono «soliti fiutare il profumo con le nari[ci] e non con la nuca e con i capelli» (Octavius, 38,2; tr. it. in Minucio Felice, 1940: 181).

Il sottile sarcasmo di Minucio Felice ci riporta però al fatto che l'atteggiamento dei primi cristiani nei confronti dei fiori non è di rifiuto verso la dimensione estetica e il piacere dei sensi che essi producono, ma si collega piuttosto all'idea che dei fiori non si debba fare un uso innaturale. Anche Tertulliano esprime lo stesso concetto quando afferma che le corone sono un "sacrilegio" contro Dio, perché con esse si fa un uso innaturale dei fiori. "La mania di cingersi la testa con dei fiori è contro natura, tanto quanto mettersi il cibo in un orecchio o voler ascoltare qualcosa con il naso» (De corona, $\mathrm{V}$, 4; tr. it. in Tertulliano 1980: 161). Trattare così i fiori è comportarsi in modo irragionevole nei confronti dei doni che Dio offre ai sensi della vista e dell'odorato.

Ancora più articolato è il ragionamento di un altro autore dello stesso periodo, Clemente Alessandrino, che considera invece l'uso di recidere i fiori come un lusso eccessivo che non si addice alla morigeratezza del cristiano. Già alcuni pagani avevano collegato l'uso dei fiori con l'ostentazione e il lusso (Goody 1993: 69-71), ma nel cristianesimo delle origini questo aspetto diventa un argomento con forte valenza apologetica perché serve a identificare la nuova religione come apportatrice di una moralità che non si trova invece nel paganesimo. Occorre ricordare che nella società romana i fiori erano collegati alla raffinatezza delle classi agiate che annoveravano tra le proprie pratiche quella di cospargere di petali i letti e i triclini e quella di bere vino 


\section{Dcula ${ }^{23}$}

Vol 21, No 23 (July 2020) • DOI: 10.12977/ocula2020-29

Graziano Lingua | Come vestono i gigli del campo. Trasformazioni dell'immaginario floreale nei primi secoli cristiani

mescolato a petali di rosa. ${ }^{5}$ Ecco perché Clemente nel Paedagogus elenca tra le cose che vanno evitate per non cadere nel lusso, non solo le vesti vistose, i profumi eccessivi e i cibi troppo elaborati, ma anche adornare il proprio corpo con fiori:

È bello in primavera sostare nei prati rugiadosi e morbidi, tappezzati di fiori svariati e nutrirsi come fanno le api di quel profumo così naturale e puro. Non è invece da persone sagge farsi una corona intrecciata [di fiori colti] dal prato intatto e portarsela a casa. (Paedagogus II, 70, 1-2; tr. it in Clemente Alessandrino 2005: 197)

\section{I gigli del campo e la cura di Dio}

L'idea di Clemente che si possa godere della bellezza dei fiori all'aperto senza doverli strappare lascia intravedere un atteggiamento diverso dal semplice rifiuto dell'uso cultuale pagano. Peraltro la prassi cristiana a questo riguardo risulta nei fatti meno rigorista di quanto non sembrerebbe emergere dai testi che abbiamo analizzato. Esistono infatti molteplici testimonianze letterarie e iconografiche che attestano come già nei primi secoli i fiori venissero utilizzati in ambito religioso sugli altari, sulle tombe dei martiri e dei semplici fedeli. ${ }^{6} \mathrm{Nel}$ IV secolo la pratica di spargere fiori sui sepolcri doveva essere particolarmente viva se Ambrogio di Milano nella sua orazione funebre per la morte di Valentiniano II si sente in dovere di protestare contro questo rito (De obitu Valentiniani consolatio, LVI, tr. it. in Ambrogio 1985: 197) o ancora Gerolamo in una delle sue lettere indirizzata a Pammachio esalta quest'ultimo perché mentre

gli altri mariti sopra la tomba delle loro mogli buttano viole, rose, gigli e fiori purpurei, cercando di dar pace al rimorso della loro coscienza con questa specie di culto [...] Pammachio, invece bagna le sante ceneri e le venerande ossa della consorte con i balsami dell'elemosina. (Epistulae, LXVI, 5; tr. it. in Gerolamo 1962: 210)

Pur manifestando un certo sospetto nei confronti dell'impiego puramente esteriore dei fiori, gli autori cristiani dei primi secoli non sono però indifferenti alla loro valenza simbolica. Gregorio di Nissa, nelle sue omelie sul Cantico dei Cantici propone ad esempio una serie di interpretazioni spirituali di fiori come il giglio, il nardo o il croco considerandoli una figura delle virtù cristiane e della vita dell'anima (Homiliae in Canticum, IV, IX; tr. it. di Gregorio di Nissa 1996: 97-99; 202-203).7 Particolarmente significativa è poi la tendenza a considerare i fiori come figura dell'amore di Dio e della bellezza del creato. A

5 A questi usi allude Tertulliano sia nel De Corona V, 4 (Tertulliano 1980, 161, n. 24), sia nel De resurrectione mortuorum, 16, 8 (Tetulliano 1990: 123).

$6 \mathrm{Al}$ riguardo si veda la voce "fleurs" in Cabrol - Leclercq, 1907-1954, Vol. 5/2.

7 Sul tema nell'opera di Gregorio di Nissa si veda Vinel (2007), saggio che videnzia come si possa registrare in questo Padre della Chiesa un embrionale interesse all'osservazione scientifica dei fiori e delle piante. 


\section{Dcula ${ }^{23}$}

Vol 21, No 23 (July 2020) • DOI: 10.12977/ocula2020-29

Graziano Lingua | Come vestono i gigli del campo. Trasformazioni dell'immaginario floreale nei primi secoli cristiani

questo riguardo i Padri potevano facilmente riferirsi all'immagine del Salmo 104 sulla bellezza caduca delle erbe e dei fiori, che gli stessi Vangeli avevano integrato nella famosa pericope sui gigli del campo.

Osservate come crescono i gigli del campo: non lavorano e non filano. Eppure io vi dico che neanche Salomone, con tutta la sua gloria vestiva come uno di loro. Ora, se Dio veste così l'erba del campo, che oggi c'è e domani si getta nel forno, non farà molto di più per voi, gente di poca fede? (Mt 6,28-29; Lc 12, 22-31)

Già lo stesso Clemente si chiedeva: «Che cosa vi è di più bello e di più rigoglioso dei fiori? Che cosa vi è di più incantevole dei gigli e delle rose». Eppure - continuava citando il passo di Mt 6, 30 - essi sono «come l'erba che oggi è nei campi e domani verrà gettata nel forno» (Paedagogus II, 103, 1; tr. it. in Clemente 2005: 227). Accanto alla dimensione della caducità nel Paedagogus la funzione simbolica dei fiori si fonda sulla possibilità di godere della loro bellezza naturale e pura (ivi, II, 70.1; tr. it.: 197), perché essi sono figura della "premura" di Dio per l'uomo (ivi, II, 76.5; tr. it.: 205).

Nel testo di Mt 6 la bellezza dei gigli era appunto collegata alla cura provvidenziale di Dio e alla necessità di non preoccuparsi troppo del futuro perché il Padre celeste, come pensa al cibo degli uccelli e come veste l'erba nella sua fugacità, così penserà anche agli uomini. Proprio in alcuni commenti a questa pericope evangelica emerge con insistenza una simbologia floreale positiva che non ha più nulla a che fare con la critica all'uso cultuale pagano. I fiori e la loro bellezza sono in questo caso espressioni della bellezza della creazione e della generosità di Dio. Ad esempio Gerolamo nel suo Exameron si lascia andare a una vera e propria esaltazione dei fiori che esprimono visivamente la perfezione del loro Creatore.

Perché descrivere le viole dal cupo colore purpureo, i candidi gigli, le rose vermiglie, le campagne tinte ora di fiori color d'oro, ora variopinti, ora color giallo zafferano [...]? Gli occhi si pascono di questa gradevole visione. [....] Quale altro artefice infatti avrebbe potuto esprimere una così grande bellezza nelle singole creature? Considerate i gigli del campo [Mt 6], quale sia il candore dei loro petali [...] se si cogliesse questo fiore e si sfogliassero i suoi petali, quale mano di artista sarebbe così abile da ridargli la forma del giglio? (Exameron, V, 8; tr. it. Ambrogio 2002: 110-111)

In questo elogio della visione dei fiori e della bellezza naturale è evidente una considerazione positiva delle forme visibili che è ormai lontana dal sospetto idolatrico in cui cadevano tutte le visibilità mondane nel comandamento veterotestamentario contro le immagini. I fiori sembrano diventare nientemeno che immagini naturali di Dio e ci riportano a quanto segnalava Goody sulla comune sorte che ha annodato il progressivo cammino di legittimazione delle immagini religiose e dei fiori come espressione materiale di realtà invisibili.

Ancora più significativo in questa direzione è il commento che Agostino fa nel De Civitate Dei del brano evangelico sui gigli del campo, dove, attraverso una vera e propria catena di rimandi che dalle forme visibili giunge fino all'in- 


\section{Dcula ${ }^{23}$}

Vol 21, No 23 (July 2020) • DOI: 10.12977/ocula2020-29

Graziano Lingua | Come vestono i gigli del campo. Trasformazioni dell'immaginario floreale nei primi secoli cristiani

visibile, presenta i fiori come una manifestazione di Dio stesso. Il vescovo di Ippona cita esplicitamente Plotino ${ }^{8}$ il quale

tratta della provvidenza trovando nella bellezza dei fiorellini e delle foglie una prova ch'essa si estende dal sommo Dio, nella sua bellezza intelligibile e ineffabile fino alle ultime realtà terrene. Tutti questi esseri, così umili e dalla vita così breve non potrebbero avere una proporzione tanto armoniosa delle forme, se non dalla forma intelligibile che possiede stabilmente in sé tutte le cose. (De civitate Dei $\mathrm{X}, 14$; tr. it. in Agostino 2001: 478-479)

La bellezza dei fiori si carica qui di una valenza ontologica tale per cui, anche se possono apparire come creature di poca importanza, partecipano secondo il loro grado all'essere divino e quindi ne sono una legittima manifestazione. Come non vedere in questo argomento di stampo neoplatonico un ragionamento simile a quello che si ritrova nelle discussioni sulla legittimità delle immagini e sulla loro capacità di manifestare l'invisibilità di Dio, attraverso la materialità delle forme visibili? Quando Dionigi l'Areopagita nel V Secolo afferma che «le bellezze visibili sono immagini della bellezza invisibile» (De coelesti hierarchia, I, 3, tr. it. in Dionigi Areopagita 1981: 79), richiama il fatto che ogni forma creaturale, anche se fragile come possono essere le erbe e i fiori, rimanda a Dio, pur appartenendo a un grado che può apparire infimo nella gerarchia degli esseri. Sarà proprio questo argomento della partecipazione tra le realtà materiali come le forme e i colori e le realtà immateriali che esse raffigurano a fare da base a una delle più importanti linee di giustificazione delle immagini religiose che confluirà nella teologia greco-bizantina delle icone.

\section{Conclusione}

Certamente i fiori non hanno goduto della stessa importanza teologica delle immagini e la loro penetrazione nella prassi cultuale cristiana appartiene più alla storia delle pratiche liturgiche che alla storia della teologia $o$ del pensiero cristiano in generale. Resta il fatto che essi sono stati un'occasione significativa per riflettere sul problema della portata rivelativa della creazione e per fare i conti con la materialità del mondo, nonché con l'importanza dei sensi nell'esperienza religiosa. Il cristianesimo delle origini ha dovuto certamente prendere le distanze da pratiche religiose che riteneva meramente esteriori e da eccessi nell'uso dei fiori che apparivano troppo distanti dal culto spirituale e dal rigore morale di cui si sentiva portatore. I fiori così sono stati al centro della polemica anti-idolatrica e dello scontro con la cultura pagana. Tuttavia gli autori cristiani non sono stati indifferenti al loro fascino e alla loro potenza simbolica e ne hanno saputo trarre occasione per riflettere su questioni che toccavano le matrici stesse di una fede

8 Agostino si riferisce a Plotino, Enneades, 3, 2, 13, 22-33 (tr. it. in Plotino 2000: $371)$. 


\section{Ocula ${ }^{23}$}

Vol 21, No 23 (July 2020) • DOI: 10.12977/ocula2020-29

Graziano Lingua | Come vestono i gigli del campo. Trasformazioni dell'immaginario floreale nei primi secoli cristiani

che avrebbe poi nei secoli successivi fatto ampio uso dei fiori non solo come elementi di decorazione, ma anche come vere e proprie immagini naturali dell'azione di Dio nel mondo.

\section{Bibliografia}

Agostino

2001 La città di Dio, trad. it. di L. Alici, Milano, Rusconi.

Ambrogio

1985 Le orazioni funebri, trad. it. a cura di G. Banterle, in Opera omnia di S. Ambrogio, Vol. 18, Roma, Città Nuova.

2002 I sei giorni della creazione, trad. it. a cura di G. Banterle, in Opera omnia di S. Ambrogio, Vol. 1, Roma, Città Nuova.

Baus, Karl

1940 Der Kranz in Antike und Christentum: eine religionsgeschichtliche Untersuchung mit besonderer Berücksichtigung Tertullians, Bonn, Hanstein.

Bremmer, Jan N.

1999 "Paradise. From Persia, via Greece, into the Septuagint", in G. Luttikhuizen (a cura di), Paradise Interpreted. Representation of Biblical Paradise in the Judaism and Christianity, Leiden, Brill, pp. 1-20.

Cabrol, Fernand; Leclercq, Henri

1907-1953 Dictionnaire d'archéologie chrétienne et de liturgie, Paris, Letouzey et Ané.

Clemente Alessandrino

2005 Il Pedagogo, trad. it. di D. Tessore, Roma, Città Nuova.

Dionigi Aeropagita

1981 Tutte le opere, tr. it. di P. Scazzoso, Milano, Rusconi.

Finkel, Irving L.

2008 "The Search for the Hanging Gardens", in Id.; M. Seymour (a cura di), Babylon. Myth and Reality, London, The British Museum Press.

Gerolamo

1962 Lettere, Vol. II, trad. it. di S. Cola, Roma, Città Nuova.

Goody, Jack

1993 La cultura dei fiori, trad. it. di M. C. Costamagna, Torino, Einaudi.

Gregorio di Nissa

1996 Omelie sul Cantico dei Cantici, trad. it. di C. Moreschini, Roma, Città Nuova.

Kelly, John N.

1984 Il pensiero cristiano delle origini, trad. it di M. Girardet, Bologna, EDB.

Menozzi, Daniele

1995 La chiesa e le immagini. I testi fondamentali sulle arti figurative dalle origini 


\section{Ocula ${ }^{23}$}

Vol 21, No 23 (July 2020) • DOI: 10.12977/ocula2020-29

Graziano Lingua | Come vestono i gigli del campo. Trasformazioni dell'immaginario floreale nei primi secoli cristiani

ai nostri giorni, Cinisello Balsamo, San Paolo.

Minucio, Felice

1940 L'Ottavio, trad. it. di P. Suffia, Roma, S.A.S.

Plotino

2000 Enneadi, trad. it. di G. Faggin, Milano, Bompiani.

Prestige, George L.

2018 Dio nel pensiero dei Padri, trad. it. di A. Comba, Bologna, EDB.

Ryken, Leland; Wilhoit, James C.; Longman III, Tremper (a cura di)

2006 Le immagini bibliche. Simboli, figure retoriche e temi letterari della Bibbia, trad. it. a cura di M. Zappella, Cinisello Balsamo, San Paolo.

Senofonte

2000 Economico, trad. it. di F. Roscalla, Milano, Rizzoli.

Tertulliano

1980 La Corona, trad. it. di P.A. Gramaglia, Roma, San Paolo.

1990 La risurrezione dei morti, trad. it. a cura di Claudio Micaelli, Roma, Città Nuova.

Tosco, Carlo

2018 Storia dei giardini. Dalla Bibbia al giardino allitaliana, Bologna, Il Mulino.

Tuplin, Christopher

1996 "The Parks and Gardens of the Achaemenid Empire", in Id., Achaemenid Studies, Stuttgart, Franz Steiner Verlag, pp. 80-131.

Vinel, Françoise

2007 “Arbres, plantes et fleurs dans l'“In Hexaéméron" et les "Homélies sur le Can $\neg$ tique des Cantiques” de Gregoire de Nysse: simples métaphores ou références scientifiques?, in La cultura scientifico-naturalistica nei Padri della Chiesa (I-V sec.), Roma, Institutum Patristicum Augustinianum, pp. 225-231.

Wilken, Robert Louis

2006 Alla ricerca del volto di Dio. La nascita del pensiero cristiano, trad. it. di S. Galli, Milano, Vita e Pensiero.

Graziano Lingua ha conseguito i dottorati in Ermeneutica e in Scienze Giuridiche presso l'Università di Torino. Attualmente è professore ordinario di Filosofia teoretica nella stessa Università. È autore, tra l'altro, di L'icona, l'idolo e la guerra delle immagini (Medusa, Milano 2006) e di Esiti della secolarizzazione (ETS, Pisa 2013). 